\title{
Glucose Metabolic Reprogramming and Cell Proliferation Arrest in Colorectal Micropapillary Carcinoma
}

\author{
Monika Vyas ${ }^{\mathrm{a}}$, Natalie Patel ${ }^{\mathrm{b}}$, Romulo Celli ${ }^{\mathrm{b}}$, Narendra Wajapeyee ${ }^{\mathrm{b}}$, \\ Dhanpat Jain ${ }^{\mathrm{b}}$, Xuchen Zhang ${ }^{\mathrm{b}, \mathrm{c}}$
}

\begin{abstract}
Background: Micropapillary carcinoma (MPC) has been reported as an aggressive variant of colorectal carcinoma (CRC) associated with frequent lymphovascular invasion and poor outcome. Altered glycogen metabolism by metabolic reprogramming plays a critical role for cancer cell growth and survival. We aimed to investigate glucose metabolic reprogramming in colorectal MPC.
\end{abstract}

Methods: Immmunostains for $\mathrm{Ki}-67$ and glucose transporter 1 (GLUT1) were performed on 10 colorectal MPCs. Real-time PCR analysis of expressions of GLUT1 and glycogen metabolizing enzymes: glycogen synthase (GYS1) and glycogen phosphorylase (PYGL) was performed on cultured monolayer and three-dimensional (3D) spheroid HCT116 colon cancer cells.

Results: GLUT1 was strongly expressed in MPC as compared to adjacent conventional glandular component, and was also significantly increased expression in 3D spheroids. Upregulation of GYS1 and PYGL was markedly increased in 3D spheroids. The proliferation rate (Ki-67) of MPC was significantly lower compared to conventional glandular component. The 3D spheroids showed increased cell cycle arrest. Our results demonstrate altered glycogen metabolism in colorectal MPC.

Conclusion: The reprogramming of glycogen metabolism in MPC provides a source of energy contributing to tumor cell survival in a low proliferation state. Targeting glucose-regulated metabolism may warrant consideration as possible MPC therapies.

Keywords: Colorectal carcinoma; Micropapillary carcinoma; Glucose; Metabolism

Manuscript submitted January 15, 2019, accepted January 30, 2019

${ }^{a}$ Department of Pathology, Memorial Sloan Kettering Cancer Center, New York, NY, USA

bDepartment of Pathology, Yale University School of Medicine, New Haven, CT, USA

${ }^{\mathrm{c} C o r r e s p o n d i n g ~ A u t h o r: ~ X u c h e n ~ Z h a n g, ~ D e p a r t m e n t ~ o f ~ P a t h o l o g y, ~ Y a l e ~ U n i-~}$ versity School of Medicine, 310 Cedar Street, PO Box 208023, New Haven, CT 06520, USA. Email: xuchen.zhang@yale.edu

doi: https://doi.org/10.14740/gr1145

\section{Introduction}

Colorectal carcinoma (CRC) is the third most commonly diagnosed cancer and the second leading cause of cancer death worldwide [1]. Micropapillary carcinoma (MPC), a subtype of $\mathrm{CRC}$, has been reported as an aggressive variant of carcinoma associated with frequent lymphovascular invasion and poor outcome [2-4]. The MPC cells (MCs) are clusters of neoplastic cells that closely adhere to each other with inverted cell polarity and are located in distinct empty spaces $[5,6]$. Such micropapillary structure can be seen with many other cancers, often constitutes one of the morphological tumor components and coexists with other histological types of cancer, or rarely it can be the only morphological pattern. Studies have shown that CRCs even with micropapillary component involving $2-5 \%$ of the tumor volume demonstrate more aggressive behavior compared to conventional CRCs $[6,7]$. However, the mechanisms by which the MCs obtain nutrients and gain aggressive biological behavior in the absence of a fibrovascular core and the formation of cell clusters with surrounding empty spaces are unclear.

Unlike normal cells with aerobic oxidation of glucose for energy supply, cancer cells possess a drastic increase in glucose uptake and glucose metabolism via anaerobic glycolysis to maintain their proliferation and survival. This metabolic reprogramming not only provides energy for cancer cells, but also provides essential macromolecules for their protein and nucleotide biosynthesis $[8,9]$. Studies show that hypoxia and tumor microenvironment play an important role in regulating cancer cell glucose metabolic reprogramming [10-12]. Hypoxia, one of the most important tumor microenvironmental changes in solid tumors, is also seen in CRCs [13]. We have shown that hypoxia-induced factor-1a (HIF-1a), a key player in the intracellular hypoxia pathway, is activated in cultured HCT116 colon cancer cells under hypoxic condition as well as in tumor budding cells of CRCs [14]. Hypoxia and associated transcription factor HIF-1a can upregulate cancer cell expression of glucose transporter 1 (GLUT1), a major glucose transporter protein in cancer cells, and induce glycogen metabolizing enzymes including glycogen synthase (GYS1) and glycogen phosphorylase (PYGL). However, the role of altered glycogen metabolism and its potential impact on the biology of MPC remain poorly understood. 


\section{Materials and Methods}

\section{MPC component assessment and immunohistochemical (IHC) stains}

Ten cases of CRC with micropapillary component involving at least $5 \%$ of the tumor volume were included in this study. The assessment of cases as MPC component was performed on H\&E-stained sections. IHC stains for GLUT1 and Ki-67 were performed on representative sections of each case. For analysis of tumor cell proliferation rate (Ki-67), three high-power fields $(\times 400)$ of MCs and the adjacent tumor cells of conventional glandular component were counted. The proliferation rate was calculated as percentage of $\mathrm{Ki}-67$ positive cells out of the total cells.

The study was approved by appropriate institutional review board.

\section{Cell culture and spheroid formation}

The HCT116 colon cancer cells were cultured in DMEM/F12 media supplemented with $10 \%$ fetal bovine serum, 100 units/ $\mathrm{mL}$ penicillin $\mathrm{G}$ and $100 \mu \mathrm{g} / \mathrm{mL}$ streptomycin at $37{ }^{\circ} \mathrm{C}$ under air $/ 5 \% \mathrm{CO}_{2}$. The cells were passed when approaching $90 \%$ confluence to provide new maintenance culture on T-75 flasks and spheroid formation in ULA 96-well round bottomed microplates. Spheroids of cells were established by simply dispensing $200 \mu \mathrm{L}$ cell suspensions at varying seeding densities, $80,000,40,000,20,000,5,000$ and 1,250 cells per well, into the ULA plate. The spheroid morphology was examined using an inverted light microscope. All spheroids used for study were from day 3 culture with 20,000 cells of seeding density per well.

\section{Cell cycle analysis}

The cultured three-dimensional (3D) spheroid and monolayer HCT116 colon cancer cells were harvested after 3-day culture and stained with propidium iodide as previously described [15]. Cell cycle distributions were determined by fluorescenceactivated cell sorter (FACS, BD Biosciences) analysis. Quantitation of the fraction of cells in different cell cycle phases was done using FlowJo software. The numbers of cells in $\mathrm{G}_{1}$ ( $2 n$ DNA), S phase $\left(>2 n\right.$ and $<4 n$ DNA) and $G_{2} / M(4 n$ DNA) were quantified, and the percent fraction of cells in each phase was calculated as previously described [14].

\section{RNA extraction and mRNA analyses}

Total RNA extraction and mRNA analyses (real-time RTPCR) were performed by using monolayer and spheroid HCT116 colon cancer cells. Primers used for human GLUT1 were sense: 5'-GGCCAAGAGTGTGCTAAAGAA-3' and anti-sense: 5'-ACAGCGTTGATGCCAGACAG-3'; GYS1 sense: 5'-GCGCTCACGTCTTCACTACTG-3' and antisense: 5'-TCCAGATGCCCATAAAAATGGC-3'; $P Y G L$ sense: 5'-CAGCCTATGGATACGGCATTC-3' and antisense: 5'-CGGTGTTGGTGTGTTCTACTTT-3'; 18S sense: 5'-CGCGGTTCTATTTTGTTGGTTT-3' and anti-sense: 5'-GCGCCGGTCCAAGAATTT-3'.

\section{Statistical analysis}

Data were expressed as mean \pm standard deviation (SD) and statistically significant difference was accepted at $\mathrm{P}<0.05$.

\section{Results}

\section{Assessment of MPC and spheroid formation in cultured colon cancer cells}

As described previously [6], CRC with micropapillary component involving at least $5 \%$ of the tumor volume was included in this study. The MPC component is characterized as small, tight cluster of neoplastic cells ( $>5$ cells) without true fibrovascular core, surrounded by an empty, cleft-like spaces that resemble small dilated lymphatic vessels $[6,16]$. The tumor cells show eosinophilic cytoplasm and pleomorphic hyperchromatic nuclei (Fig. 1a, b).

In contrast to most traditional cell culture systems, a 3D cell culture with spheroid formation mimicking MPC in vivo has been used to study tumor invasion, metastasis and drug sensitivity [17, 18]. We used HCT116 human colon cancer cells and cultured as monolayer (Fig. 1c) or 3D spheroids (Fig. 1d). As seen in Fig. 1d, the 3D spheroids morphologically simulate cell clusters in MPC.

\section{MPC and cultured spheroid colon cancer cells show in- creased GLUT1 expression}

The mechanisms by which MPC obtains nutrients in the absence of a fibrovascular core and the formation of cell clusters with surrounding empty spaces are unclear. Altered cellular glycogen metabolism by metabolic reprogramming of cancer cells plays a critical role for cell growth and promotes cancer cell survival. GLUT1 has a high affinity for glucose, and is the glucose transporter that is most often upregulated across other cancer types. High expression of GLUT1 correlates with disease progression and poor survival $[19,20]$. We investigated if there is increased GLUT1 expression in MCs compared to that of conventional tumor cells from the main tumor. GLUT1 was strongly expressed in MCs as compared to adjacent tumor cells of conventional glandular component in all the $10 \mathrm{CRC}$ cases (Fig. 2a). Similar to MCs in CRCs, GLUT1 expression was significantly increased in cultured 3D spheroids compared to monolayer HCT116 colon cancer cells (Fig. 2b). These data indicate that the increase of cytoplasmic glucose transportation through upregulation of GLUT1 exists in both in vivo CRC MCs and in vitro cultured 


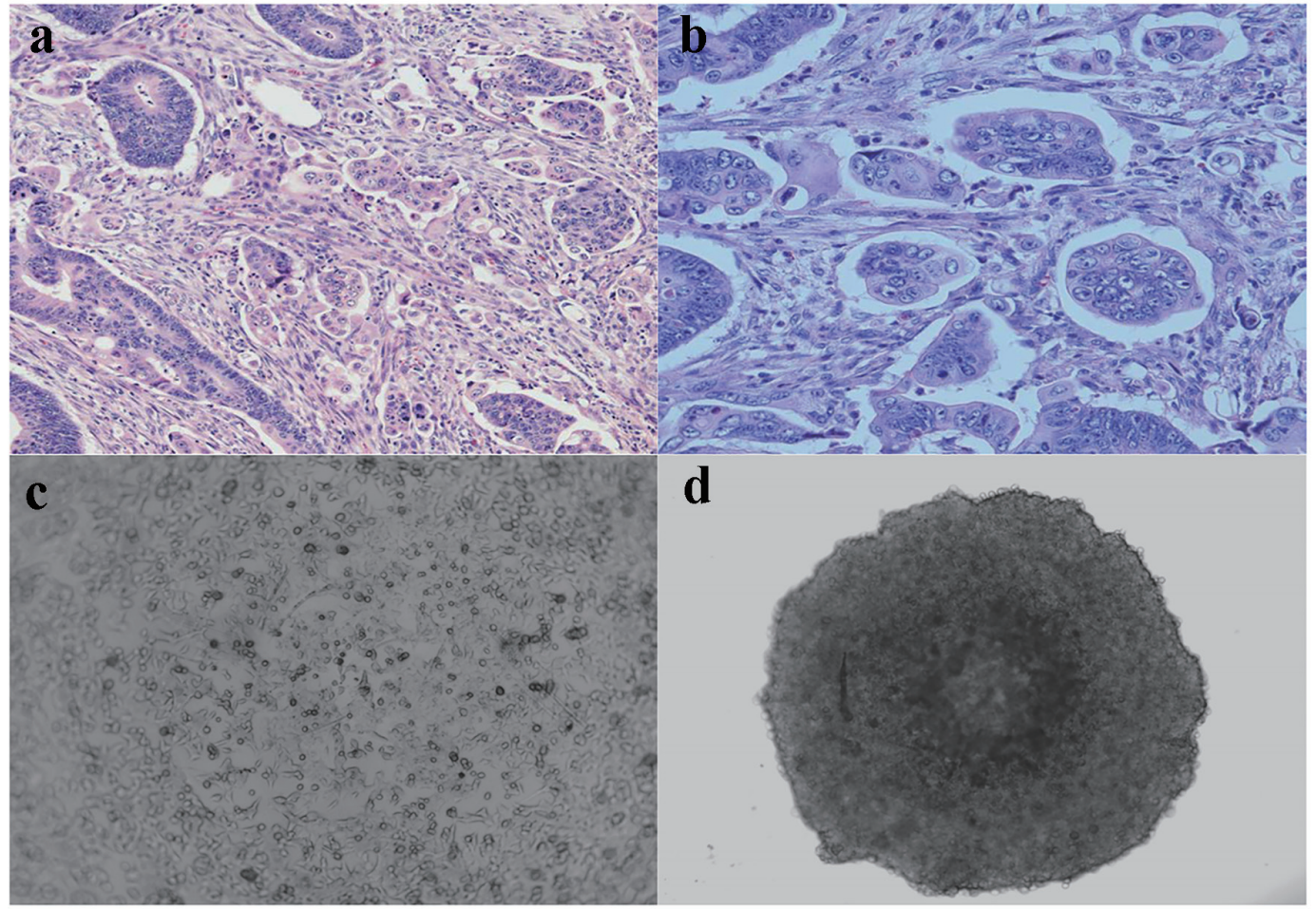

Figure 1. Micropapillary carcinoma and spheroid formation. (a) Characteristic cell clusters surrounded by empty spaces (H\&E, $\times 100)$. (b) Micropapillary cells displaying a high grade of anaplasia (H\&E, $\times 400)$. (c) Cultured monolayer HCT116 human colon cancer cells (phase contrast, $\times 200$ ). (d) 3D spheroid HCT116 human colon cancer cells formed in ULA round-bottom microplate (phase contrast, $\times 200)$.

3D spheroids.

Spheroid colon cancer cells show significantly increased expression of glycogen metabolizing enzymes

We have shown hypoxic microenvironment exists at the ad- vancing tumor front in CRCs [14]. Glycogen accumulation under hypoxic conditions has been observed in several cancer cell lines and studies have suggested a critical role of glycogen in promoting survival of cancer cells [21, 22]. Synthesis and breakdown of glycogen involves the activity of several enzymes and regulatory proteins. Among these, GSY1 and PYGL catalyze the key steps of glycogen synthesis and deg-

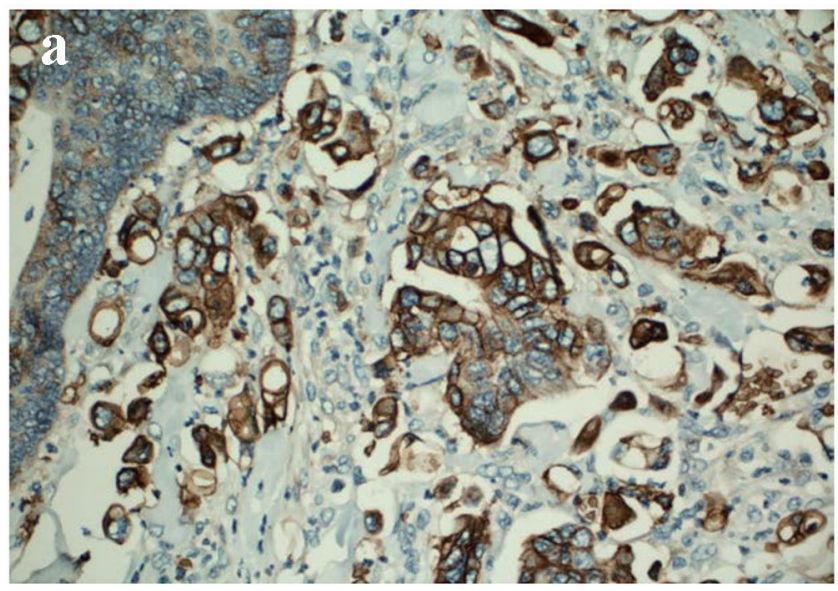

b

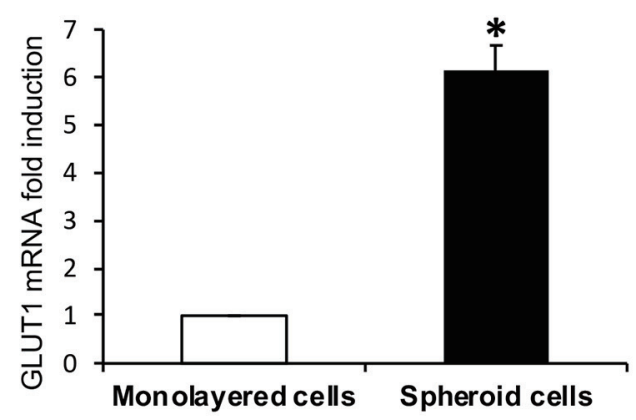

Figure 2. GLUT1 expression. (a) Tumor cells of micropapillary component showing increased immunohistochemical expression of GLUT1 compared to adjacent tumor cells of conventional glandular component (DAB, × 200). (b) GLUT1 expression is significantly increased in 3D spheroids than that of monolayer HCT116 colon cancer cells assayed by real-time RT-PCR (sixfold induction, $\left.{ }^{*} \mathrm{P}<0.01\right)$. 

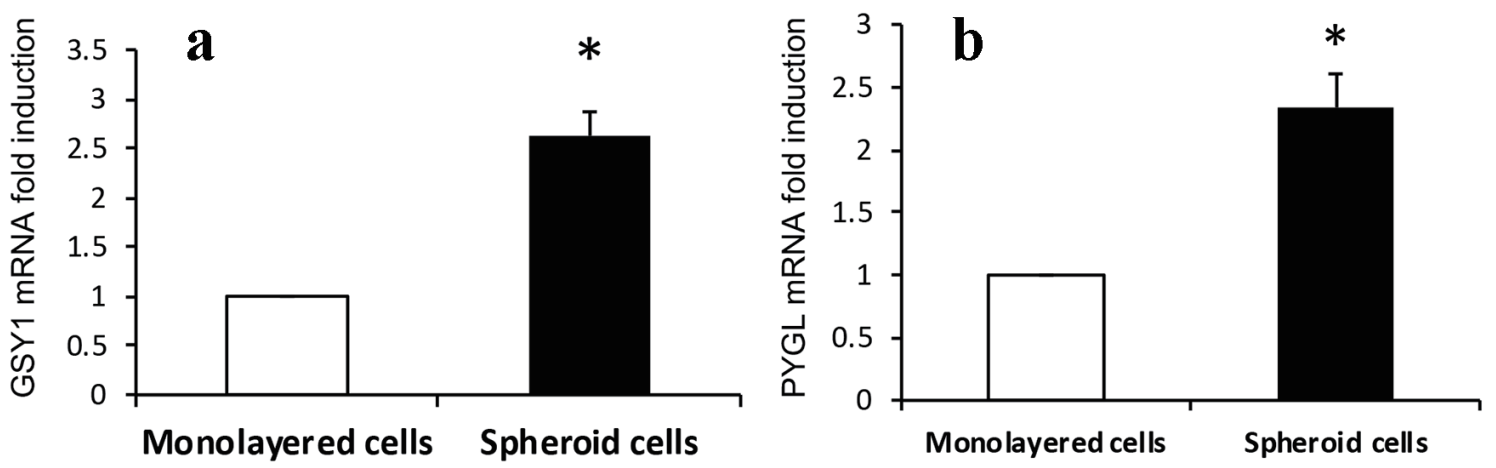

Figure 3. Expression of (a) GSY1 and (b) PYGL. Expressions of GSY1 and PYGL are significantly increased in 3D spheroids than that of monolayer HCT116 colon cancer cells assayed by real-time RT-PCR (2.6- and 2.3-fold induction, respectively, ${ }^{*} \mathrm{P}<$ $0.01)$.

radation, respectively $[20,23]$. We investigated if there is increased expression of glycogen metabolizing enzymes GYS1 and PYGL in cultured 3D spheroids. As seen in Fig. 3a and b, the expression of two fundamental glycogen metabolizing enzymes GYS1 and PYGL was markedly increased in 3D spheroids as compared to monolayer HCT116 colon cancer cells.

\section{MPS shows cell proliferation arrest}

Although it is well accepted that high proliferation index is associated with tumor progression, lymphovascular invasion and metastasis, we have identified tumor budding cells in CRCs, one important aggressive histomorphology, showed significantly decreased proliferation index compared to adjacent tumor cells of conventional glandular component assessed by $\mathrm{Ki}-67 \mathrm{IHC}$ [14]. We then investigated if there is decreased proliferation rate of MCs as seen in tumor budding cells. The
MCs in CRCs showed significantly lower proliferation rate (Ki-67 positive cells) compared to adjacent tumor cells of conventional glandular component (Fig. 4a, b). Similarly, the cultured 3D spheroids showed significant increase of cells in G0/ G1 phase compared to monolayer HCT116 colon cancer cells $(59.50 \pm 2.35 \%$ vs. $20.87 \pm 0.67 \%, \mathrm{P}<0.001)$ (Fig. $4 \mathrm{c}$ ).

\section{Discussion}

Carcinoma with micropapillary pattern or MPC has been recognized as a distinct histologic type and described in various organs including lung, breast, urinary bladder, stomach and others. Studies have demonstrated that, irrespective of organ of origin, the presence of micropapillary pattern in carcinomas is closely associated with lymph node or distant metastases, lymphovascular invasion, local recurrence and worse prognosis with shorter survival [24-28]. MPC of colorectum was first
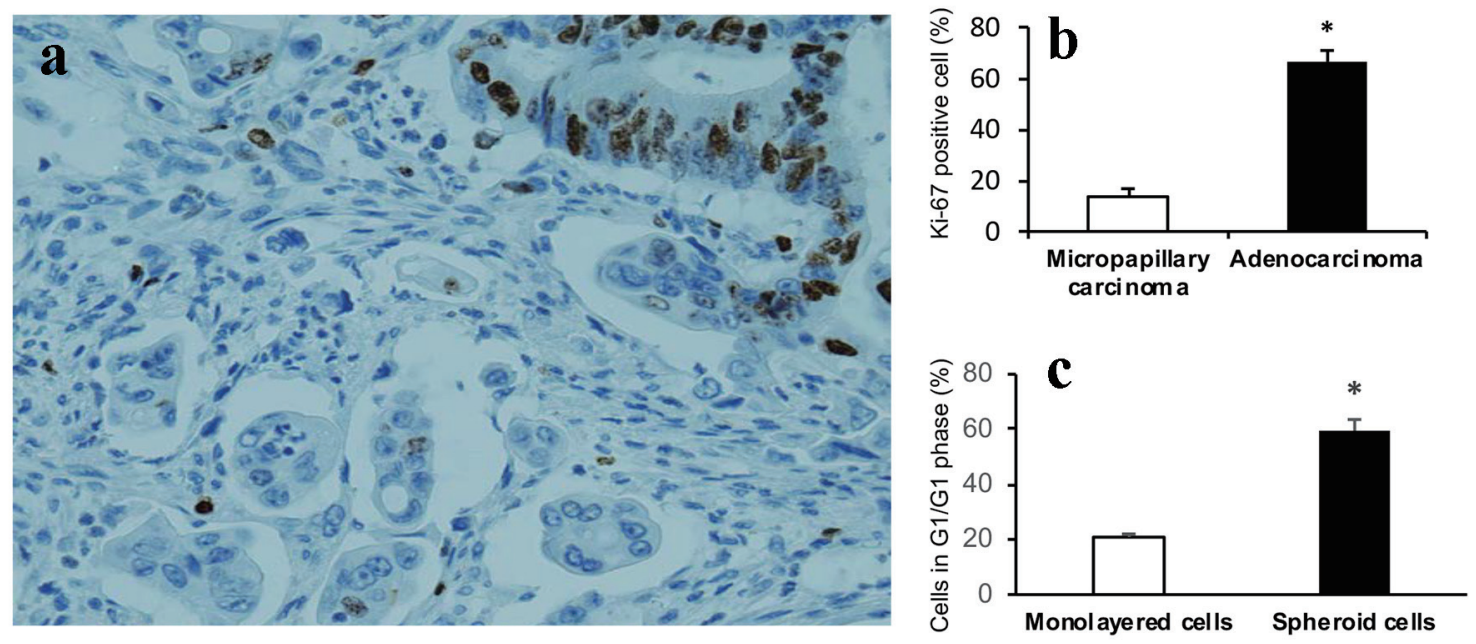

Figure 4. Cell proliferation arrest. (a) Tumor cells of micropapillary component showing significant lower proliferation rate compared to adjacent tumor cells of conventional glandular component assessed by Ki-67 (nuclear expression) (DAB, $\times 400)$. (b) Graphical quantitation of Ki-67 positive cells expressed as percent of total cells. Data are expressed as mean \pm SD. ${ }^{*} P<0.001$. (c) Cells in G0/G1 phase are significantly increased in 3D spheroids than that of monolayer HCT116 colon cancer cells. Data are expressed as mean $\pm \mathrm{SD}$. ${ }^{*} \mathrm{P}<0.001$ 
reported by Sakamoto et al in 2005 [29]. To date, the reported CRC with MCs accounts for 9-19\% of all CRCs [4, 6, 30-32]. Similar to MPC in other organs, colorectal MPC has been associated with a higher stage, frequent lymphovascular invasion, lymph node metastasis and distant metastasis, and poor clinical outcomes compared with conventional adenocarcinoma $[30,32]$. The proportion of an MPC component needed for the diagnosis of MPC has not yet been decided, whereas special variants of $\mathrm{CRC}$ such as mucinous adenocarcinoma or signet ring cell carcinoma are designated such, if more than $50 \%$ of the lesion is composed of mucin or signet ring cells. Currently, most studies using micropapillary component involving $2-5 \%$ of the tumor volume have demonstrated the aggressive biologic behavior, which is irrespective of the percentage of the MPC component [7, 30]. Therefore, recognition of MPC component is important as MPC appears to be an aggressive variant of CRC.

Although CRC with MPC pattern behaves aggressively even in TNM stage I and II, and has higher proportion of TP53 mutations $[6,28]$, the mechanisms underlying this aggressive biologic behavior is still unclear. Histologically, MPC is characterized by small clusters of cohesive neoplastic cells without fibrovascular cores floating in empty spaces. The empty spaces are lined by fibrocollagenous stroma without vascular endothelial lining. Although it has been assumed that these empty spaces are retraction artifact resulting from tissue fixation and processing, a study has confirmed that they are real in vivo and are likely related to altered tumor-stromal interactions but not an artifactual phenomenon [33]. The presence of empty spaces and absence of fibrovascular cores raise the question that how the MCs obtain nutrients in a hypoxic microenvironment and gain the aggressive biologic behavior. To sustain survival and continuous replication, cancer cells must reprogram their metabolism and nutrient acquisition to support their energy, synthesis and redox needs [34]. We have demonstrated previously that hypoxic microenvironment exists in tumor front and tumor budding cells in CRCs. Hypoxia-exposed cultured colon cancer cells show cell cycle arrest and low $\mathrm{Ki}-67$ proliferation rate [14]. These data indicate that hypoxic microenvironment may contribute to the decreased proliferation rate of CRC tumor budding cells. Similarly, MPC showed lower proliferation rate (Ki-67) and the cultured 3D spheroids mimicking MPC in vitro showed cell cycle arrest as well. The lower proliferation rate and cell cycle arrest in G0/G1 status may facilitate MCs survival by reducing energy consumption in a hypoxic microenvironment.

Glycogen accumulation under hypoxic conditions has been observed in several cancer cell lines. Studies have suggested a critical role of increased glycogen uptake through glucose transporter GLUT1 in promoting the survival of cancer cells $[21,22]$. Furthermore, increased synthesis and breakdown of glycogen in cancer cells through regulation of glycogen synthase and glycogen phosphorylase play important roles in cancer cell survival in a status of oxygen deprivation $[23,35]$. Exposure of U87 glioblastoma, MCF-7 breast and HCT116 colon cancer cells to hypoxia-induced expression of two major glycogen metabolism enzymes, namely GYS1 and the liver isoform of glycogen phosphorylase PYGL, and loss of PYGL in these tumor cell lines resulted in tumor cell senes- cence and cell death [23]. In our current study, we demonstrated significant increase of GLUT1 expression in both MPC and cultured 3D spheroids. We also identified that the expression of two fundamental glycogen metabolizing enzymes GYS1 and PYGL was markedly increased in 3D spheroids. These data indicate that the reprogramming of glycogen metabolism in MPC provides a source of energy contributing to the tumor cell survival. Like other targeted therapies being developed, anticancer agents targeted on GLUT1 and glucose metabolism associated enzymes have demonstrated promising anticancer activities [36-38]. Targeting inhibition of these glycogen metabolism enzymes might warrant consideration as possible anticancer therapies in future studies for colorectal MPCs.

In conclusion, our results demonstrate that MPC and the cultured 3D spheroids showed upregulated GLUT1 expression and cell cycle arrest. Expressions of glycogen metabolizing enzymes GYS1 and PYGL were markedly increased in 3D spheroids as compared to monolayer HCT116 cells. The cultured 3D spheroid formation may be used to study the biologic behavior, metabolic alteration and possible target therapy on $\mathrm{MPC}$ in vitro.

\section{Acknowledgments}

This manuscript was partially presented in the 2016 and 2017 USCAP annual meetings.

\section{Financial Support}

None.

\section{Conflict of Interest}

The authors have no conflict of interest to disclose.

\section{Author Contributions}

All authors have read and approved the final version of the present manuscript.

\section{References}

1. Bray F, Ferlay J, Soerjomataram I, Siegel RL, Torre LA, Jemal A. Global cancer statistics 2018: GLOBOCAN estimates of incidence and mortality worldwide for 36 cancers in 185 countries. CA Cancer J Clin. 2018;68(6):394424.

2. Guzinska-Ustymowicz K, Niewiarowska K, Pryczynicz A. Invasive micropapillary carcinoma: a distinct type of adenocarcinomas in the gastrointestinal tract. World J Gastroenterol. 2014;20(16):4597-4606.

3. Hisamori S, Nagayama S, Kita S, Kawamura J, Yoshizawa A, Sakai Y. Rapid progression of submucosal in- 
vasive micropapillary carcinoma of the colon in progressive systemic sclerosis: report of a case. Jpn J Clin Oncol. 2009;39(6):399-405.

4. Lino-Silva LS, Salcedo-Hernandez RA, Caro-Sanchez $\mathrm{CH}$. Colonic micropapillary carcinoma, a recently recognized subtype associated with histological adverse factors: clinicopathological analysis of 15 cases. Colorectal Dis. 2012;14(9):e567-572.

5. Cserni G. Reversed polarity of the glandular epithelial cells in micropapillary carcinoma of the large intestine and the EMA/MUC1 immunostain. Pathology. 2014;46(6):527-532.

6. Verdu M, Roman R, Calvo M, Rodon N, Garcia B, Gonzalez M, Vidal A, et al. Clinicopathological and molecular characterization of colorectal micropapillary carcinoma. Mod Pathol. 2011;24(5):729-738.

7. Jakubowska K, Guzinska-Ustymowicz K, Pryczynicz A. Invasive micropapillary component and its clinicohistopathological significance in patients with colorectal cancer. Oncol Lett. 2016;12(2):1154-1158.

8. Vander Heiden MG, Cantley LC, Thompson CB. Understanding the Warburg effect: the metabolic requirements of cell proliferation. Science. 2009;324(5930):1029-1033.

9. Pavlova NN, Thompson CB. The emerging hallmarks of cancer metabolism. Cell Metab. 2016;23(1):27-47.

10. Huang D, Li C, Zhang H. Hypoxia and cancer cell metabolism. Acta Biochim Biophys Sin (Shanghai). 2014;46(3):214-219.

11. Xie H, Simon MC. Oxygen availability and metabolic reprogramming in cancer. J Biol Chem. 2017;292(41):16825-16832.

12. Sun L, Suo C, Li ST, Zhang H, Gao P. Metabolic reprogramming for cancer cells and their microenvironment: Beyond the Warburg Effect. Biochim Biophys Acta Rev Cancer. 2018;1870(1):51-66.

13. Dekervel J, Hompes D, van Malenstein H, Popovic D, Sagaert X, De Moor B, Van Cutsem E, et al. Hypoxiadriven gene expression is an independent prognostic factor in stage II and III colon cancer patients. Clin Cancer Res. 2014;20(8):2159-2168.

14. Vyas M, Patel N, Nagarajan A, et al. Hypoxia induced HIF-1 alpha expression promotes angiogenesis, tumor budding cell survival and cell proliferation arrest in highgrade tumor budding colorectal carcinomas. Int J Clin Exp Patho. 2016;9:13047-13055.

15. Nagarajan A, Dogra SK, Liu AY, Green MR, Wajapeyee N. PEA15 regulates the DNA damage-induced cell cycle checkpoint and oncogene-directed transformation. Mol Cell Biol. 2014;34(12):2264-2282.

16. Lugli A, Kirsch R, Ajioka Y, Bosman F, Cathomas G, Dawson H, El Zimaity H, et al. Recommendations for reporting tumor budding in colorectal cancer based on the International Tumor Budding Consensus Conference (ITBCC) 2016. Mod Pathol. 2017;30(9):1299-1311.

17. Wen Z, Liao Q, Hu Y, You L, Zhou L, Zhao Y. A spheroidbased 3-D culture model for pancreatic cancer drug testing, using the acid phosphatase assay. Braz J Med Biol Res. 2013;46(7):634-642.
18. Doublier S, Belisario DC, Polimeni M, Annaratone L, Riganti C, Allia E, Ghigo D, et al. HIF-1 activation induces doxorubicin resistance in MCF7 3-D spheroids via P-glycoprotein expression: a potential model of the chemo-resistance of invasive micropapillary carcinoma of the breast. BMC Cancer. 2012;12:4.

19. Ancey PB, Contat C, Meylan E. Glucose transporters in cancer - from tumor cells to the tumor microenvironment. FEBS J. 2018;285(16):2926-2943.

20. Ritterson Lew C, Guin S, Theodorescu D. Targeting glycogen metabolism in bladder cancer. Nat Rev Urol. 2015;12(7):383-391.

21. Shukla SK, Mulder SE, Singh PK. Hypoxia-Mediated In Vivo Tumor Glucose Uptake Measurement and Analysis. Methods Mol Biol. 2018;1742:107-113.

22. Zois CE, Harris AL. Glycogen metabolism has a key role in the cancer microenvironment and provides new targets for cancer therapy. J Mol Med (Berl). 2016;94(2):137154.

23. Favaro E, Bensaad K, Chong MG, Tennant DA, Ferguson DJ, Snell C, Steers G, et al. Glucose utilization via glycogen phosphorylase sustains proliferation and prevents premature senescence in cancer cells. Cell Metab. 2012;16(6):751-764.

24. Dai C, Xie H, Kadeer X, Su H, Xie D, Ren Y, She Y, et al. Relationship of Lymph Node Micrometastasis and Micropapillary Component and Their Joint Influence on Prognosis of Patients With Stage I Lung Adenocarcinoma. Am J Surg Pathol. 2017;41(9):1212-1220.

25. Wu Y, Zhang N, Yang Q. The prognosis of invasive micropapillary carcinoma compared with invasive ductal carcinoma in the breast: a meta-analysis. BMC Cancer. 2017;17(1):839.

26. Bertz S, Wach S, Taubert H, Merten R, Krause FS, Schick $\mathrm{S}$, Ott OJ, et al. Micropapillary morphology is an indicator of poor prognosis in patients with urothelial carcinoma treated with transurethral resection and radiochemotherapy. Virchows Arch. 2016;469(3):339-344.

27. Eom DW, Kang GH, Han SH, Cheon GJ, Han KH, Oh HS, Kim JH, et al. Gastric micropapillary carcinoma: A distinct subtype with a significantly worse prognosis in TNM stages I and II. Am J Surg Pathol. 2011;35(1):8491.

28. Xu F, Xu J, Lou Z, Di M, Wang F, Hu H, Lai M. Micropapillary component in colorectal carcinoma is associated with lymph node metastasis in T1 and T2 Stages and decreased survival time in TNM stages I and II. Am J Surg Pathol. 2009;33(9):1287-1292.

29. Sakamoto K, Watanabe M, De La Cruz C, Honda H, Ise H, Mitsui K, Namiki K, et al. Primary invasive micropapillary carcinoma of the colon. Histopathology. 2005;47(5):479-484.

30. Kim MJ, Hong SM, Jang SJ, Yu E, Kim JS, Kim KR, Gong $\mathrm{G}$, et al. Invasive colorectal micropapillary carcinoma: an aggressive variant of adenocarcinoma. Hum Pathol. 2006;37(7):809-815.

31. Lee HJ, Eom DW, Kang GH, Han SH, Cheon GJ, Oh HS, $\mathrm{Han} \mathrm{KH}$, et al. Colorectal micropapillary carcinomas are 
associated with poor prognosis and enriched in markers of stem cells. Mod Pathol. 2013;26(8):1123-1131.

32. Haupt B, Ro JY, Schwartz MR, Shen SS. Colorectal adenocarcinoma with micropapillary pattern and its association with lymph node metastasis. Mod Pathol. 2007;20(7):729-733.

33. Acs G, Paragh G, Chuang ST, Laronga C, Zhang PJ. The presence of micropapillary features and retraction artifact in core needle biopsy material predicts lymph node metastasis in breast carcinoma. Am J Surg Pathol. 2009;33(2):202-210.

34. DeBerardinis RJ, Chandel NS. Fundamentals of cancer metabolism. Sci Adv. 2016;2(5):e1600200.

35. Pescador N, Villar D, Cifuentes D, Garcia-Rocha M, Or-
tiz-Barahona A, Vazquez S, Ordonez A, et al. Hypoxia promotes glycogen accumulation through hypoxia inducible factor (HIF)-mediated induction of glycogen synthase 1. PLoS One. 2010;5(3):e9644.

36. Shahruzaman SH, Fakurazi S, Maniam S. Targeting energy metabolism to eliminate cancer cells. Cancer Manag Res. 2018;10:2325-2335.

37. Wang G, Wang JJ, Yin PH, Xu K, Wang YZ, Shi F, Gao $\mathrm{J}$, et al. New strategies for targeting glucose metabolismmediated acidosis for colorectal cancer therapy. J Cell Physiol. 2018;234(1):348-368.

38. Shi Y, Liu S, Ahmad S, Gao Q. Targeting key transporters in tumor glycolysis as a novel anticancer strategy. Curr Top Med Chem. 2018;18(6):454-466. 